\title{
A mesoscopic approach to diffusion phenomena in mixtures
}

\author{
Nunzia Palumbo ${ }^{1}$, Christina Papenfusss,*, and Patrizia Rogolino' \\ ${ }^{1}$ Department of Mathematics, University of Messina, 98166 Messina, Italy \\ 2 Technische Universität Berlin, Institut für Mechanik, Straße des 17. Juni 135, \\ D-10623 Berlin, Germany \\ *Corresponding author (c.papenfuss@gmx.de) \\ Communicated by W. Muschik, Berlin, Germany and K.-H. Hoffmann, Chemnitz, \\ Germany
}

\begin{abstract}
The mesoscopic concept is applied to the theory of mixtures. The aim is to investigate the diffusion phenomenon from a mesoscopic point of view. The domain of the field quantities is extended by the set of mesoscopic variables, here the velocities of the components. Balance equations on this enlarged space are the equations of motion for the mesoscopic fields. Moreover, local distribution functions of the velocities are introduced as a statistical element, and an equation of motion for this distribution function is derived. From this equation of motion, differential equations for the diffusion fluxes and also for higher order fluxes are obtained. These equations are of balance type, as it is postulated in extended thermodynamics. The resulting evolution equation for the diffusion flux generalizes Fick's law.
\end{abstract}

\section{Introduction}

Most phenomena involving mass diffusion are described by means of Fick's law, which relates linearly the mass flow to the gradient of the mass concentration through the diffusion coefficient. In practical applications, Fick's law is satisfactory to a large extent. However, it is not applicable to transitory situations involving for instance high frequencies. The mass balance together with Fick's law leads to parabolic differential equations for the concentrations, allowing for infinite velocities of propagation of disturbances. The last paradox must be overcome by considering non-Fickian transport behavior 
for the diffusive mass flux. A possible solution is given in extended thermodynamics [1-5]. The diffusion flux is introduced as an additional field variable, obeying a differential equation. This differential equation is derived from an irreversible thermodynamics treatment $[6,7]$ of the dissipation inequality (for other applications see [8-13]), or a balance type equation for the nonconvective fluxes, here the diffusion flux, is postulated in rational extended thermodynamics $[5,14,15]$. For a purely macroscopic derivation of balance type differential equations for higher order fluxes see [16, 17]. Within rational extended thermodynamics, it has been shown that the resulting system of field equations is symmetric hyperbolic with a convex extension $[5,18,19]$, thereby allowing for finite speeds of disturbances only [18, 20, 21].

A foundation of the field equations of extended thermodynamics has been given in statistical mechanics. In most cases, the single particle distribution function is used, together with the Boltzmann equation [5]. In this case microscopic interactions are introduced via the collision term in the Boltzmann equation. However, the Boltzmann equation is, strictly speaking, valid only for dilute gases, and for liquids or solids a different foundation is necessary. Such a background theory can be given using the BBGKY hierarchy [22-25] for the hierarchy of N-particle distribution functions. The structure of the field equations of extended thermodynamics could be derived from this statistical mechanics background. Such an approach necessitates the knowledge of microscopic interparticle interaction potentials.

An approach, different from microscopic statistical mechanics, is the socalled mesoscopic theory $[26,29,30]$. It is a continuum theory, introducing constitutive functions on a continuum level, and no microscopic interparticle interactions. On the other hand, the mesoscopic description is more detailed than the macroscopic description. This additional information leads to the definition of internal variables in complex media, and to equations of motion for them. This mesoscopic concept has been applied to liquid crystals [27, 31$36]$, to damage and fracture mechanics [37, 38], and to dipolar media [28]. We will show in the following that within the mesoscopic theory a balance type equation for the diffusion flux can be derived.

The basic idea of the mesoscopic theory is to introduce an additional variable, the so-called mesoscopic variable, in the domain of the field quantities. On this higher dimensional space a continuum theory is developed. In this theory the mesoscopic field quantities depend not only on position and time, but also on the mesoscopic variable. In addition, a local distribution function of the mesoscopic variable is introduced as a statistical element. Macroscopic fields are the averages of mesoscopic ones over the additional (mesoscopic) variable. 
The aim of this paper is the application of the mesoscopic concept to an Ncomponent mixture and a derivation of differential equations for the diffusion fluxes from the mesoscopic theory. The application of the resulting equations to fast phenomena involving diffusion processes is left for future work, as well as the investigation whether our generalized diffusion equation leads to hyperbolic field equations.

\section{Mesoscopic concept and balance equations}

The mesoscopic concept introduces the mesoscopic space,

$$
(\boldsymbol{m}, \mathbf{x}, t) \in \mathscr{M} \times \mathbb{R}_{x}^{3} \times \mathbb{R}_{t},
$$

on which all field quantities are defined. Here $\boldsymbol{m}$ is a set of mesoscopic variables which is an element of a suitable manifold $\mathscr{M}$ on which an integration can be defined. Beyond the use of additional variables, $\mathbf{m}$ the mesoscopic concept introduces a statistical element, the so-called mesoscopic distribution function (MDF) $f(\mathbf{m}, \mathbf{x}, t)$ generated by the different values of the mesoscopic variable of the particles in a volume element,

$$
f(\mathbf{m}, \mathbf{x}, t) \equiv f(\cdot), \quad(\cdot) \equiv(\mathbf{m}, \mathbf{x}, t) \in \mathscr{M} \times R_{x}^{3} \times R_{t} .
$$

The MDF describes the distribution of $\mathbf{m}$ in a volume element around $\mathbf{x}$ at time $t$, and therefore it is normalized:

$$
\int f(\mathbf{m}, \mathbf{x}, t) d \mathbf{m}=1
$$

It is the probability density of having the particular value $\mathbf{m}$ of the mesoscopic variable in the continuum element at position $\mathbf{x}$ and time $t$. Therefore it is the fraction

$$
f(\mathbf{m}, \mathbf{x}, t)=\frac{\rho(\mathbf{m}, \mathbf{x}, t)}{\rho(\mathbf{x}, t)} .
$$

Here $\rho(\mathbf{x}, t)$ is the macroscopic mass density. By use of Eq. (2) we obtain

$$
\rho(\mathbf{x}, t)=\int \rho(\mathbf{m}, \mathbf{x}, t) d \mathbf{m} .
$$

\subsection{Balance equations}

Let $G$ denote a region in $\mathbb{R}^{3} \times \mathscr{M}$ and $\boldsymbol{X}$ the density of an extensive quantity. Then the global quantity in the region $G$ changes due to a flux over the boundary of $G$ and due to production and supply within $G$ : 


$$
\frac{d}{d t} \int_{G} \boldsymbol{X} d^{3} x d \boldsymbol{m}=\int_{\partial G} \boldsymbol{\phi}_{\boldsymbol{X}}(\cdot) d a+\int_{G} \Sigma_{X}(\cdot) d^{3} x d \boldsymbol{m} .
$$

A generalized Reynolds transport theorem in the mesoscopic space, analogous to the one in [39], is used to transform the time derivative, and Gauss theorem is applied to the boundary integral. The boundary $\partial G$ of $G$ consists of a boundary in position space and a boundary on the manifold $\mathscr{M}$. Then we have in regular points of the continuum the local mesoscopic balance [40]:

$$
\frac{\partial}{\partial t} \boldsymbol{X}(\cdot)+\nabla_{x} \cdot[\mathbf{v}(\cdot) \boldsymbol{X}(\cdot)-\boldsymbol{S}(\cdot)]+\nabla_{m} \cdot[\boldsymbol{w}(\cdot) \boldsymbol{X}(\cdot)-\boldsymbol{R}(\cdot)]=\boldsymbol{\Sigma}(\cdot)
$$

Here the independent field $\boldsymbol{w}(\cdot)$, defined on the mesoscopic space, describes the change in time of the set of mesoscopic variables: With respect to $\boldsymbol{m}$ the mesoscopic change velocity $\boldsymbol{w}(\cdot)$ is the analogue to the mesoscopic material velocity $\mathbf{v}(\cdot)$ referring to $\boldsymbol{x}$ : If a particle is characterized by $(\boldsymbol{m}, \boldsymbol{x}, t)$, then for $\Delta t \rightarrow+0$ it is characterized by $(\boldsymbol{m}+\boldsymbol{w}(\cdot) \Delta t, \boldsymbol{x}+\mathbf{v}(\cdot) \Delta t, t+\Delta t)$ at later times $t+\Delta t$. Besides the usual gradient, also the gradient with respect to the set of mesoscopic variables appears. This expresses the fact that there is a flux over the boundary on the manifold $\mathscr{M}$.

According to Eq. (2) we obtain from the mesoscopic mass balance a balance type differential equation for the $\operatorname{MDF} f(\cdot)$ by inserting its definition [30, 41]:

$$
\begin{array}{r}
\frac{\partial}{\partial t} f(\cdot)+\nabla_{x} \cdot[\mathbf{v}(\cdot) f(\cdot)]+\nabla_{m} \cdot[\boldsymbol{w}(\cdot) f(\cdot)] \\
\quad+f(\cdot)\left[\frac{\partial}{\partial t}+\mathbf{v}(\cdot) \cdot \nabla_{x}\right] \ln \varrho(\boldsymbol{x}, t)=0
\end{array}
$$

\section{Application of the mesoscopic concept to mixtures}

Let us consider a mixture of $\mathrm{N}$ different chemical components. The different components are distinguished by a component index in capital letters. We will introduce velocity distributions of all chemical components, denoted $f^{A}$. Each of them is a function of position of the respective continuum element, time, and particle velocity. The function $f^{A}(\mathbf{x}, t, \mathbf{v})$ gives the probability density of finding the value $\mathbf{v}$ for the material velocity of component $A$ of the mixture in a volume element around $\mathbf{x}$ at time $t$.

The mesoscopic mass densities and all other mesoscopic fields are different for different chemical components. Mesoscopic fields are denoted by a "hat", where this is necessary in order to distinguish them from the corresponding macroscopic quantities. 
The distribution function $f^{A}$ is defined as follows:

$$
f^{A}(\mathbf{x}, t, \mathbf{v})=: \frac{\hat{\rho}^{A}(\mathbf{x}, t, \mathbf{v})}{\rho^{A}(\mathbf{x}, t)}
$$

$f^{A}(\mathbf{x}, t, \mathbf{v}) d \mathbf{v}$ gives the fraction of particles of component $A$ having a material velocity in the element $d \mathbf{v}$ on $\mathbb{R}_{v}^{3}$ around $\mathbf{v}$.

The distribution functions are normalized:

$$
\int_{\mathbb{R}_{v}^{3}} f^{A}(\mathbf{x}, t, \mathbf{v}) d \mathbf{v}=1, \quad \forall A
$$

\section{Macroscopic balance equations for the mixture and its components}

In this section, we shall recall briefly the macroscopic balance equations for mixtures and for the different components.

The total mass of a mixture is a conserved quantity, while the mass of a particular component is not conserved if chemical reactions occur. The same happens for momentum, and energy due to chemical reactions between different components of the mixture. Therefore, in the balance equations for components there are production terms on the right-hand side.

\subsection{Macroscopic balance equations for the mixture}

The balance equations for the mixture as a whole look the same as the balance equations for a one-component system [42, 43]. From the structure of balance laws, mixtures cannot be distinguished from chemically pure substances.

\section{Balance of mass}

$$
\frac{\partial \rho}{\partial t}(\mathbf{x}, t)+\nabla \cdot(\rho \mathbf{v})(\mathbf{x}, t)=0
$$

Here $\mathbf{v}$ is the macroscopic material velocity of the mixture, i.e., the barycentric velocity of all particles in the volume element.

\section{Balance of momentum}

$$
\frac{\partial}{\partial t}(\rho \mathbf{v})+\nabla \cdot\left(\rho \mathbf{v} \otimes \mathbf{v}-\mathbf{t}^{T}\right)=\rho \mathbf{f}
$$


where $\mathbf{t}$ denotes Cauchy stress tensor, and $\mathbf{f}$ is the acceleration (specific density of volume forces).

\section{Balance of energy}

$$
\frac{\partial}{\partial t}\left[\rho\left(e+\frac{1}{2} \mathbf{v} \cdot \mathbf{v}\right)\right]+\nabla \cdot\left[\mathbf{q}-\mathbf{v} \cdot \mathbf{t}+\rho \mathbf{v}\left(e+\frac{1}{2} \mathbf{v} \cdot \mathbf{v}\right)\right]-\rho(\mathbf{f} \cdot \mathbf{v}+r)=0,
$$

where $e$ is the specific internal energy density (internal energy per unit mass), q denotes the heat flux density, and $r$ is the energy supply density.

\subsection{Macroscopic balance equations for the components}

\section{Balance of mass of component $A$}

The local balance of mass of component $A$, taking into account production of mass of that component due to chemical reactions, reads:

$$
\frac{\partial \rho^{A}}{\partial t}(\mathbf{x}, t)+\nabla \cdot\left(\rho^{A} \mathbf{v}^{A}\right)(\mathbf{x}, t)=P_{\text {chem }}^{A},
$$

where $\mathbf{v}^{A}$ is the material velocity of component $A$. The mass densities of the different chemical components are additive:

$$
\sum_{A} \rho^{A}=\rho
$$

The material velocity $\mathbf{v}$ of the mixture is defined as the weighted sum of the component velocities:

$$
\sum_{A} \rho^{A} \mathbf{v}^{A}=\rho \mathbf{v}
$$

Equation (13) may be cast in a more useful form in terms of mass fractions $c^{A}$ defined by $c^{A}=\frac{\rho^{A}}{\rho}, A=1, \ldots, N$ and the diffusion fluxes $\mathbf{J}^{A}$,

$$
\mathbf{J}^{A}=\rho^{A}\left(\mathbf{v}^{A}-\mathbf{v}\right),
$$

such that $\sum_{A} \mathbf{J}^{A}=0$. The mass fraction balance equation is

$$
\rho \frac{d}{d t} c^{A}+\nabla \cdot \mathbf{J}^{A}=P_{c h e m}^{A},
$$

where $\frac{d}{d t}=\frac{\partial}{\partial t}+\mathbf{v} \cdot \nabla$ is the material time derivative. 


\section{Balance of momentum of component $A$}

The momentum balance for one particular chemical component is:

$$
\frac{\partial}{\partial t}\left(\rho^{A} \mathbf{v}^{A}\right)+\nabla \cdot\left(\rho^{A} \mathbf{v}^{A} \otimes \mathbf{v}^{A}-\mathbf{t}^{A^{T}}\right)=\rho^{A} \mathbf{f}^{A}+\mathbf{P}_{m}^{A},
$$

with a production $P_{m}^{A}$ of momentum of component $A$.

\section{Balance of energy of component $A$}

The energy balance equation is given by

$$
\begin{gathered}
\frac{\partial}{\partial t}\left[\rho^{A}\left(e^{A}+\frac{1}{2} \mathbf{v}^{A} \cdot \mathbf{v}^{A}\right)\right]+\nabla \cdot\left[\mathbf{q}^{A}-\mathbf{v}^{A} \cdot \mathbf{t}^{A}+\rho^{A} \mathbf{v}^{A}\left(e^{A}+\frac{1}{2} \mathbf{v}^{A} \cdot \mathbf{v}^{A}\right)\right] \\
-\rho^{A}\left(\mathbf{f}^{A} \cdot \mathbf{v}^{A}+r^{A}\right)=P_{e}^{A},
\end{gathered}
$$

with a component energy production due to chemical reactions. In this set of balance equations, constitutive equations for the component stress tensor, component internal energy density, and heat flux density, and the productions due to chemical reactions are needed in order to close the system of differential equations. These constitutive quantities must be related to the wanted fields in a material-dependent manner. The constitutive theory, including restrictions on constitutive functions from general principles, is out of the scope of the present paper.

The (total) energy density of the mixture is the sum over the components. From the additivity of the extensive quantities mass, momentum, and energy follows that the balances of the mixture are obtained summing up the component equations $[42,43]$. This leads to relations between constitutive quantities for components and constitutive quantities for the mixture.

\section{Mesoscopic balance equations for the mixture and its components}

Mesoscopic balance equations are defined on the mesoscopic space. For the mixture as a whole the additional mesoscopic variable is the material velocity $\mathbf{v}$ of the mixture, which is the weighted sum of the component velocities, see Eq. (15). The mesoscopic balance equations are derived analogously to the derivation discussed in the second section (see [6] for the general structure of these balances) and by suitable identifications. The mesoscopic balance equations for the mixture are those derived previously for the one component system (see Section 2). In all cases it is supposed that the constituents of the 
mixture have no internal angular momentum, i.e., we are not dealing with micropolar continua.

For the mixture we introduce the distribution function of the material velocity:

$$
f(\boldsymbol{x}, t, \mathbf{v})=f(\bullet)=\frac{\hat{\rho}(\bullet)}{\rho(\boldsymbol{x}, t)}
$$

Explicitly we have the following mesoscopic balances:

\subsection{Mesoscopic balance equations for the mixture}

Mesoscopic balance of mass

$$
\frac{\partial}{\partial t}(\hat{\rho})(\bullet)+\nabla \cdot(\hat{\rho} \mathbf{v})(\bullet)+\nabla_{\mathbf{v}} \cdot(\mathbf{w} \hat{\rho})(\bullet)=0
$$

where $(\bullet)$ is the abbreviation for $(\boldsymbol{x}, t, \mathbf{v}), \mathbf{v}$ and $\mathbf{w}$ are the mesoscopic velocity of the mixture and the change in time of the mesoscopic variable $\mathbf{v}$, respectively $(\mathbf{w}=\dot{\mathbf{v}})$.

According to the definition of the distribution function Eq. (20), we obtain from the mesoscopic mass balance (21) a differential equation for the MDF $f(\bullet)$ :

$$
\frac{\partial f(\bullet)}{\partial t}+\nabla \cdot(f(\bullet) \mathbf{v})+\nabla_{\mathbf{v}}(f(\bullet) \mathbf{w}(\bullet))+f(\bullet)\left(\frac{\partial}{\partial t}+\mathbf{v} \cdot \nabla\right) \lg \rho(\mathbf{x}, t)=0
$$

\section{Mesoscopic balance of momentum}

$$
\frac{\partial}{\partial t}(\hat{\rho} \mathbf{v})+\nabla \cdot(\hat{\rho} \mathbf{v} \otimes \mathbf{v})+\nabla_{\mathbf{v}} \cdot(\hat{\rho} \mathbf{w} \otimes \mathbf{v})-\nabla \cdot \hat{\boldsymbol{t}}^{T}-\nabla_{\mathbf{v}} \cdot \hat{\mathbf{T}}^{T}=\hat{\rho} \hat{\mathbf{f}},
$$

with $\hat{\boldsymbol{t}}^{T}=\hat{\boldsymbol{t}}$ and $\hat{\boldsymbol{T}}^{T}=\hat{\boldsymbol{T}}$. Here $\hat{\boldsymbol{T}}$ is the analogue in velocity space of the stress tensor, i.e., the nonconvective momentum flux in velocity space. All field quantities here depend on position, time and particle velocity.

As a result of definition (20), the macroscopic material velocity of the mixture, $\mathbf{v}(\mathbf{x}, t)$, i.e., the mean velocity of the mixture, is expressed as follows:

$$
\rho(\mathbf{x}, t) \mathbf{v}(\mathbf{x}, t)=\int_{\mathbb{R}^{3}} \hat{\rho} \mathbf{v} d \mathbf{v}
$$




\section{Mesoscopic balance of energy}

$$
\begin{aligned}
\frac{\partial}{\partial t}\left[\hat{\rho}\left(\hat{e}+\frac{1}{2} \mathbf{v} \cdot \mathbf{v}\right)\right]+\nabla \cdot\left[\hat{\mathbf{q}}-\mathbf{v} \cdot \hat{\mathbf{t}}+\rho\left(\hat{e}+\frac{1}{2} \mathbf{v} \cdot \mathbf{v}\right) \mathbf{v}\right] \\
\quad+\nabla_{\mathbf{v}} \cdot\left[\hat{\mathbf{Q}}-\mathbf{w} \cdot \hat{\mathbf{T}}+\hat{\rho} \mathbf{w}\left(\hat{e}+\frac{1}{2} \mathbf{v} \cdot \mathbf{v}\right)\right]=\hat{\rho}(\hat{\mathbf{f}} \cdot \mathbf{v}+\hat{r})+\hat{P}_{e}
\end{aligned}
$$

$\mathbf{Q}$ is the analogue to the heat flux density in velocity space, i.e., a nonconvective energy flux over the boundary in the mesoscopic part of the domain, which consists of position, time and velocity for all fields here.

By integrating these mesoscopic balances over the mesoscopic space, i.e., over all velocities, with the mesoscopic distribution function as statistical weight, the macroscopic balance equations for the mixture are obtained.

\subsection{Mesoscopic balance equations for the components of the mixture}

\section{Mesoscopic balance of mass of component A}

By using the general form of the mesoscopic balance of mass (see also [30, 33, $41,44])$, we have the following equation for the component A:

$$
\frac{\partial}{\partial t}\left(\hat{\rho}^{A}\right)(\cdot)+\nabla \cdot\left(\hat{\rho}^{A} \mathbf{v}^{A}\right)(\cdot)+\nabla_{\mathbf{v}} \cdot\left(\mathbf{w}^{A} \hat{\rho}^{A}\right)(\cdot)=\hat{P}_{\text {chem }}^{A}(\cdot),
$$

where $\mathbf{v}^{A}$ represents the material velocity of component $A$, and $\mathbf{w}^{\mathbf{A}}$ is the change in time of the mesoscopic variable $\mathbf{v}^{A}: \mathbf{w}^{\mathbf{A}}=\dot{\mathbf{v}}^{\mathbf{A}}$. This acceleration $\mathbf{w}^{\mathbf{A}}$ is a mesoscopic constitutive function. The term on the right hand side represents a production due to chemical reactions.

The sum of the mass balances of the different components is the mass balance of the mixture, because the mass densities are additive:

$$
\begin{aligned}
& \sum_{A=1, \ldots, N} \hat{\rho}^{A}=\hat{\rho}, \\
& \frac{\partial}{\partial t} \sum_{A}\left(\hat{\rho}^{A}\right)+\nabla \cdot \sum_{A}\left(\hat{\rho}^{A} \mathbf{v}^{A}\right)+\nabla_{\mathbf{v}} \cdot \sum_{A}\left(\mathbf{w}^{A} \hat{\rho}^{A}\right)=\sum_{A} \hat{P}_{\text {chem }}^{A} .
\end{aligned}
$$

By comparing in Eqs. (28) and (21) the fluxes in mesoscopic space and in position space, we obtain the following relations: 


$$
\begin{aligned}
& \sum_{A} \nabla_{\mathbf{v}} \cdot\left(\mathbf{w}^{A} \hat{\rho}^{A}\right)=\nabla_{\mathbf{v}} \cdot(\mathbf{w} \hat{\rho}), \\
& \sum_{A} \nabla \cdot\left(\hat{\rho}^{A} \mathbf{v}^{A}\right)=\nabla \cdot(\hat{\rho} \mathbf{v}),
\end{aligned}
$$

and for the production terms,

$$
\sum_{A} \hat{P}_{\text {chem }}^{A}=0
$$

A solution of Eqs. (29) and (30) is

$$
\begin{aligned}
& \mathbf{w}=\sum_{A} \frac{\hat{\rho}^{A}}{\hat{\rho}} \mathbf{w}^{A}, \\
& \mathbf{v}=\sum_{A} \frac{\hat{\rho}^{A}}{\hat{\rho}} \mathbf{v}^{A} .
\end{aligned}
$$

\section{Mesoscopic distribution function for the components}

The distribution function $f^{A}(\mathbf{x}, t, \mathbf{v})$ gives the probability density of finding a particle of component $A$ with velocity $\mathbf{v}^{A}$ in the volume element around $\mathbf{x}$ at time $t$. It is the mass fraction

$$
f^{A}(\mathbf{x}, t, \mathbf{v})=\frac{\hat{\rho}^{A}(\mathbf{x}, t, \mathbf{v})}{\rho^{A}(\mathbf{x}, t)}
$$

The mesoscopic balance of mass for component $A$, Eq. (26), can be used to derive an equation of motion for the distribution function:

$$
\begin{aligned}
\frac{\partial f^{A}}{\partial t} & +\nabla \cdot\left(f^{A} \mathbf{v}^{A}\right)+\nabla_{\mathbf{v}} \cdot\left(f^{A} \mathbf{w}^{A}\right) \\
& +f^{A}\left(\frac{\partial}{\partial t}+\mathbf{v}^{A} \cdot \nabla\right) \lg \rho^{A}(\mathbf{x}, t)=\frac{\hat{P}_{\text {chem }}^{A}}{\rho^{A}(\mathbf{x}, t)}
\end{aligned}
$$

Introducing the macroscopic fields by integrating over the mesoscopic part of the enlarged domain, here the velocity, with the component distribution function as probability density, we can transform local mesoscopic balances into 
local macroscopic balances. So, the definition of the macroscopic material velocity of the component $A, \mathbf{v}^{A}(\mathbf{x}, t)$, i.e., the mean velocity of the component $A$ of the mixture, is

$$
\rho^{A} \mathbf{v}^{A}(\mathbf{x}, t)=\int_{\mathbb{R}^{3}} \hat{\rho}^{A} \mathbf{v} d \mathbf{v}
$$

and Eq. (26) leads to the macroscopic mass balance of component $A$ (13) if we take

$$
\int \nabla_{\mathbf{v}} \cdot\left[\mathbf{w}^{A} \hat{\rho}^{A}\right] d \mathbf{v}=0
$$

into consideration. This holds because of Gauss's theorem and because $f^{A}(\cdot)$, restricted to the mesoscopic part, has a compact support.

Relation between the mesoscopic distribution functions of the components and the distribution function of the mixture

The velocity distribution function of a particular component $f^{A}$ was defined as

$$
f^{A}(\mathbf{x}, t, \mathbf{v})=\frac{\hat{\rho}^{A}(\mathbf{x}, t, \mathbf{v})}{\rho^{A}(\mathbf{x}, t)}
$$

and the velocity distribution of the mixture is

$$
f(\mathbf{x}, t, \mathbf{v})=\frac{\hat{\rho}(\mathbf{x}, t, \mathbf{v})}{\rho(\mathbf{x}, t)} .
$$

Because the mass densities of the different chemical components are additive,

$$
\sum_{A} \hat{\rho}^{A}(\mathbf{x}, t, \mathbf{v})=\hat{\rho}(\mathbf{x}, t, \mathbf{v}), \quad \sum_{A} \rho^{A}(\mathbf{x}, t)=\rho(\mathbf{x}, t),
$$

we end up with a relation between the distribution functions of the components and that of the mixture:

$$
\sum_{A} f^{A}(\mathbf{x}, t, \mathbf{v}) \rho^{A}(\mathbf{x}, t)=\sum_{A} \hat{\rho}^{A}(\mathbf{x}, t, \mathbf{v})=\hat{\rho}(\mathbf{x}, t, \mathbf{v})=f(\mathbf{x}, t, \mathbf{v}) \rho(\mathbf{x}, t)
$$


or

$$
\sum_{A} f^{A}(\mathbf{x}, t, \mathbf{v}) \frac{\rho^{A}(\mathbf{x}, t)}{\rho(\mathbf{x}, t)}=f(\mathbf{x}, t, \mathbf{v})
$$

The distribution function of the mixture is the sum of the component distribution functions, weighted with the mass fractions of the components.

\section{Mesoscopic balance of momentum of component A}

$$
\begin{gathered}
\frac{\partial}{\partial t}\left(\hat{\rho}^{A} \mathbf{v}^{A}\right)+\nabla \cdot\left(\hat{\rho}^{A} \mathbf{v}^{A} \otimes \mathbf{v}^{A}\right)+\nabla_{\mathbf{v}} \cdot\left(\hat{\rho}^{A} \mathbf{w}^{A} \otimes \mathbf{v}^{A}\right) \\
-\nabla \cdot \hat{\mathbf{t}}^{A}-\nabla_{\mathbf{v}} \cdot \hat{\mathbf{T}}^{A}=\hat{\rho}^{A} \hat{\mathbf{F}}^{A}+\hat{\mathbf{P}}_{m}^{A}
\end{gathered}
$$

The mesoscopic momenta $\hat{\rho}^{A} \mathbf{v}^{A}$ are additive, and summing up Eq. (43) over the different components one obtains the mesoscopic balance of momentum (23). By comparing the fluxes in position space and in velocity space, respectively, we have

$$
\begin{aligned}
& \hat{\mathbf{t}}=\sum_{A}\left(\hat{\mathbf{t}}^{A}-\hat{\rho}^{A} \delta \mathbf{v}^{A} \otimes \delta \mathbf{v}^{A}\right), \\
& \hat{\mathbf{T}}=\sum_{A}\left(\hat{\mathbf{T}}^{A}-\hat{\rho}^{A} \delta \mathbf{w}^{A} \otimes \delta \mathbf{v}^{A}\right),
\end{aligned}
$$

where we have introduced the abbreviations

$$
\delta \mathbf{v}^{A}=\mathbf{v}^{A}-\mathbf{v}, \quad \delta \mathbf{w}^{A}=\mathbf{w}^{A}-\mathbf{w} .
$$

Finally, a comparison of the production terms in the mesoscopic balances of momentum for the sum of components on one hand, and for the mixture on the other hand, leads to the relation

$$
\hat{\rho} \hat{\mathbf{f}}=\sum_{A}\left(\hat{\rho}^{A} \hat{\mathbf{f}}^{A}+\hat{\mathbf{P}}_{m}^{A}\right) .
$$

By integrating over the mesoscopic space the macroscopic momentum balance for component $A$, Eq. (18) is obtained. 


\section{Mesoscopic balance of energy of component A}

For particles of component $A$ and particle velocity $\mathbf{v}^{A}$ we have

$$
\begin{aligned}
\frac{\partial}{\partial t}\left[\hat{\rho}^{A}\right. & \left.\left(\hat{e}^{A}+\frac{1}{2} \mathbf{v}^{A} \cdot \mathbf{v}^{A}\right)\right]+\nabla \cdot\left[\hat{\mathbf{q}}^{A}-\mathbf{v}^{A} \cdot \hat{\mathbf{t}}^{A}+\rho^{A}\left(\hat{e}^{A}+\frac{1}{2} \mathbf{v}^{A} \cdot \mathbf{v}^{A}\right) \mathbf{v}^{A}\right] \\
& +\nabla_{\mathbf{v}} \cdot\left[\hat{\mathbf{Q}}^{A}-\mathbf{w}^{A} \cdot \hat{\mathbf{T}}^{A}+\hat{\rho}^{A} \mathbf{w}^{A}\left(\hat{e}^{A}+\frac{1}{2} \mathbf{v}^{A} \cdot \mathbf{v}^{A}\right)\right] \\
= & \hat{\rho}^{A}\left(\hat{\mathbf{f}}^{A} \cdot \mathbf{v}^{A}+\hat{r}^{A}\right)+\hat{P}_{e}^{A} .
\end{aligned}
$$

As before, by summing over all components of the mixture one obtains the mesoscopic balance of energy (25), and the integration over $\mathbb{R}^{3}$ leads to the macroscopic balance of energy expressed by Eq. (12).

\section{Constitutive quantities for the components compared to those for the mixture}

Analogously to the previous section, the flux terms and the production terms in the balance equations of the mesoscopic mixture and the sum of the component equations are compared. As a result the internal energy density of the mixture, the heat flux and its analogue in mesoscopic space, and the energy absorption density of the mixture are expressed by the following relations:

$$
\begin{aligned}
& \hat{\rho} \hat{e}=\sum_{A}\left(\hat{\rho}^{A} \hat{\boldsymbol{e}}^{A}+\frac{1}{2} \hat{\rho}^{A} \delta \mathbf{v}^{A} \cdot \delta \mathbf{v}^{A}\right) \\
& \hat{\mathbf{q}}=\sum_{A}\left\{\hat{\mathbf{q}}^{A}-\delta \mathbf{v}^{A} \cdot \hat{\mathbf{t}}^{A}+\hat{\rho}^{A} \hat{\boldsymbol{e}}^{A} \delta \mathbf{v}^{A}+\frac{1}{2}\left[\hat{\rho}^{A}\left(\delta \mathbf{v}^{A} \cdot \delta \mathbf{v}^{A}\right) \delta \mathbf{v}^{A}\right]\right\}, \\
& \hat{\mathbf{Q}=} \sum_{A}\left\{\hat{\mathbf{Q}}^{A}-\hat{\rho}^{A}\left(\mathbf{w} \cdot \delta \mathbf{w}^{A}\right) \delta \mathbf{v}^{A}-\delta \mathbf{w}^{A} \cdot \hat{\mathbf{T}}^{A}+\hat{\rho}^{A} \hat{e}^{A} \delta \mathbf{w}^{A}\right. \\
& \left.\quad+\hat{\rho}^{A}\left(\delta \mathbf{v}^{A} \cdot \mathbf{v}\right) \delta \mathbf{w}^{A}+\frac{1}{2}\left[\hat{\rho}^{A}\left(\delta \mathbf{v}^{A} \cdot \delta \mathbf{v}^{A}\right) \delta \mathbf{w}^{A}\right]\right\} \\
& \hat{\rho} \hat{r}=\sum_{A}\left(\hat{\rho}^{A} \hat{r}^{A}+\hat{\rho}^{A} \mathbf{v}^{A} \cdot \delta \hat{\mathbf{f}}^{A}\right) \quad \text { with } \delta \hat{\mathbf{f}}^{A}=\hat{\mathbf{f}}^{A}-\hat{\mathbf{f}}, \\
& \hat{P_{e}=}=\sum_{A} \hat{P}_{e}^{A} .
\end{aligned}
$$

Equations (49) to (53) show that the mesoscopic constitutive quantities of the mixture are not, in general, the sum of the corresponding constitutive quantities of the components, but some fluctuation terms contribute. 


\section{Evolution equations for diffusion fluxes}

The aim of this section is to obtain evolution equations for diffusion fluxes of the different components. By using relations (24), (36) and the definitions (8), (20) it is possible to define the diffusion flux $\mathbf{J}^{A}$, expressed by equation (16), as follows:

$$
\mathbf{J}^{A}(\mathbf{x}, t)=\rho^{A}(\mathbf{x}, t) \int_{\mathbb{R}^{3}}\left[f^{A}(\cdot)-f(\cdot)\right] \mathbf{v} d \mathscr{M},
$$

where integration is over the mesoscopic variable $\mathbf{v} \in \mathscr{M}$.

In order to derive evolution equations for fluxes let us introduce the family of the macroscopic fields of order parameters which is defined by different moments of the distribution functions $f^{A}(\cdot)$ and $f(\cdot)$. They are defined as

$$
\begin{aligned}
& \mathbf{a}_{k}^{A}=\int_{\mathbb{R}^{3}} f^{A}(\cdot) \underbrace{\overline{\mathbf{v} \ldots \mathbf{v}}}_{k} d \mathscr{M}, \\
& \mathbf{a}_{k}=\int_{\mathbb{R}^{3}} f(\cdot) \underbrace{\mathbf{v} \ldots \mathbf{v}}_{k} d \mathscr{M},
\end{aligned}
$$

where $\ldots$ denotes the symmetric irreducible (traceless) part of a tensor [33]. These fields of order parameters describe macroscopically the mesoscopic state of the system introduced by $\mathbf{v}^{A}$ and $\mathbf{v}$ and its distribution functions $f^{A}$ and $f$. Thus these are the link between the mesoscopic background description of the system and its extended description by additional macroscopic fields. In a macroscopic phenomenological theory, they represent in general internal variables which satisfy relaxation equations. In our case, the first moments $\mathbf{a}_{1}$ and $\mathbf{a}_{1}^{A}$ are not internal variables, but classical wanted fields, namely the specific momentum densities (material velocities) $\mathbf{v}$ and $\mathbf{v}^{A}$. The higher order moments are internal variables in the sense of thermodynamics.

The diffusion fluxes $\mathbf{J}^{A}(A=1, \ldots, N)$ are proportional to the difference of the first moment of the distribution functions $f^{A}(\cdot)$ and $f(\cdot)$. If we multiply Eqs. (35) and (22) with the mesoscopic variable and integrate over the manifold $\mathscr{M}$, here the velocity space, we obtain, respectively,

$$
\begin{aligned}
\int_{\mathbb{R}^{3}} & \frac{\partial}{\partial t}\left[f^{A}(\cdot) \mathbf{v}^{A}\right] d \mathscr{M}+\int_{\mathbb{R}^{3}} \nabla \cdot\left[\mathbf{v}^{A} f^{A}(\cdot) \mathbf{v}^{A}\right] d \mathscr{M} \\
& +\int_{\mathbb{R}^{3}} \mathbf{v}^{A} \nabla_{\mathbf{v}} \cdot\left[\mathbf{w}^{A}(\cdot) f^{A}(\cdot)\right] d \mathscr{M} \\
& +\int_{\mathbb{R}^{3}} f^{A} \mathbf{v}^{A}\left[\frac{\partial}{\partial t}+\mathbf{v} \cdot \nabla\right] \lg \rho^{A}(\mathbf{x}, t) d \mathscr{M}=\int_{\mathbb{R}^{3}} \frac{\hat{P}_{\text {chem }}^{A}}{\rho^{A}(\mathbf{x}, t)} d \mathscr{M}
\end{aligned}
$$


and

$$
\begin{aligned}
\int_{\mathbb{R}^{3}} & \frac{\partial}{\partial t}[f(\cdot) \mathbf{v}] d \mathscr{M}+\int_{\mathbb{R}^{3}} \nabla \cdot[\mathbf{v} f(\cdot) \mathbf{v}] d \mathscr{M} \\
& \quad+\int_{\mathbb{R}^{3}} \mathbf{v} \nabla_{\mathbf{v}} \cdot[\mathbf{w}(\cdot) f(\cdot)] d \mathscr{M}+\int_{\mathbb{R}^{3}} f \mathbf{v}\left[\frac{\partial}{\partial t}+\mathbf{v} \cdot \nabla\right] \lg \rho(\mathbf{x}, t) d \mathscr{M}=0 .
\end{aligned}
$$

The mesoscopic manifold $\mathscr{M}$, here the $\mathbb{R}^{3}$ spanned by the velocities $\mathbf{v}$, is time independent. The time derivative and the derivative with respect to position can be interchanged with the integration over $\mathscr{M}$. We split the mesoscopic velocity into the macroscopic velocity and the deviation from this average, respectively, for component $\mathrm{A}$ and the mixture,

$$
\mathbf{v}(\cdot)=\mathbf{v}^{A}(\mathbf{x}, t)+\delta \mathbf{v}^{A}
$$

and

$$
\mathbf{v}(\cdot)=\mathbf{v}(\mathbf{x}, t)+\delta \mathbf{v}
$$

The resulting equations of motion for the first moments $\mathbf{a}_{1}^{A}$ and $\mathbf{a}_{1}$ are, respectively,

$$
\begin{aligned}
& \frac{\partial}{\partial t} \mathbf{a}_{1}^{A}+\nabla \cdot\left[\mathbf{v}^{A}(\mathbf{x}, t) \mathbf{a}_{1}^{A}+\int_{\mathbb{R}^{3}} \delta \mathbf{v}^{A}(\cdot) f^{A}(\cdot) \mathbf{v}^{A} d \mathscr{M}\right] \\
& \quad+\int_{\mathbb{R}^{3}} \mathbf{v}^{A} \nabla_{\mathbf{v}} \cdot\left[f^{A}(\cdot) \mathbf{w}^{A}(\cdot)\right] d \mathscr{M}+\mathbf{a}_{1}^{A}\left[\frac{\partial}{\partial t}+\mathbf{v}^{A}(\mathbf{x}, t) \cdot \nabla\right] \lg \rho^{A}(\mathbf{x}, t) \\
& \quad+\nabla \lg \rho^{A}(\mathbf{x}, t) \int_{\mathbb{R}^{3}} \delta \mathbf{v}_{A}(\cdot) f^{A}(\cdot) \mathbf{v}^{A} d \mathscr{M}=\frac{1}{\rho^{A}(\mathbf{x}, t)} \int \hat{P}_{\text {chem }}^{A} \mathbf{v}^{A} d \mathscr{M}
\end{aligned}
$$

and

$$
\begin{aligned}
\frac{\partial}{\partial t} \mathbf{a}_{1} & +\nabla \cdot\left[\mathbf{v}(\mathbf{x}, t) \mathbf{a}_{1}+\int_{\mathbb{R}^{3}} \delta \mathbf{v}(\cdot) f(\cdot) \mathbf{v} d \mathscr{M}\right] \\
& +\int_{\mathbb{R}^{3}} \mathbf{v} \nabla_{\mathbf{v}} \cdot[f(\cdot) \mathbf{w}(\cdot)] d \mathscr{M}+\mathbf{a}_{1}\left[\frac{\partial}{\partial t}+\mathbf{v}(\mathbf{x}, t) \cdot \nabla\right] \lg \rho(\mathbf{x}, t) \\
& +\nabla \lg \rho(\mathbf{x}, t) \int_{\mathbb{R}^{3}} \delta \mathbf{v}(\cdot) f(\cdot) \mathbf{v} d \mathscr{M}=0 .
\end{aligned}
$$

We now introduce some approximations: the deviations $\delta \mathbf{v}^{A}$ and $\delta \mathbf{v}$ vanish, i.e., the mesoscopic variable $\mathbf{v}$ has the value of the barycentric velocity. In other words, we suppose $\mathbf{v}^{A}(\cdot)=\mathbf{v}^{A}(\mathbf{x}, t)$ and $\mathbf{v}(\cdot)=\mathbf{v}(\mathbf{x}, t)$. 
By computing the difference of Eqs. (60) and (61) and taking into account these approximations, one has:

$$
\begin{gathered}
\frac{\partial}{\partial t}\left(\mathbf{a}_{1}^{A}-\mathbf{a}_{1}\right)+\nabla \cdot\left(\mathbf{v}^{A} \mathbf{a}_{1}^{A}-\mathbf{v a} \mathbf{a}_{1}\right)+\int_{\mathbb{R}^{3}} \mathbf{v} \nabla_{\mathbf{v}} \cdot f^{A}(\cdot) \mathbf{w}^{A}(\cdot) \\
-f(\cdot) \mathbf{w}(\cdot) d \mathscr{M}+\mathbf{a}_{1}^{A}\left[\frac{\partial}{\partial t}+\mathbf{v}^{A}(\mathbf{x}, t) \cdot \nabla\right] \lg \rho^{A}(\mathbf{x}, t) \\
-\mathbf{a}_{1}\left[\frac{\partial}{\partial t}+\mathbf{v}(\mathbf{x}, t) \cdot \nabla\right] \lg \rho(\mathbf{x}, t)=\frac{1}{\rho(\mathbf{x}, t)} \int_{\mathbb{R}^{3}} \hat{P}_{c h e m}^{A} d \mathscr{M} .
\end{gathered}
$$

The last equation (62) represents a general phenomenological evolution equation for diffusion fluxes in an "extended theory". Using further approximations, it is possible to obtain a Cattaneo-type diffusion equation. In order to exploit Eq. (62) further, it is necessary to insert expressions for $\mathbf{w}$ and $\mathbf{w}^{A}$. These are equations on the mesoscopic level, and they can be interpreted as mesoscopic constitutive equations. In this case, the domain of constitutive mappings, the state space has to be introduced. In mesoscopic theories there are different possibilities: one is to introduce a state space of purely mesoscopic variables, another one is to introduce a mixed space including mesoscopic and macroscopic variables, and the third possibility is one containing only macroscopic variables. The mesoscopic background of the constitutive theory has been applied to liquid crystals of uniaxial molecules [34, 41, 45, 46] and to dipolar media [28]. For diffusion in mixtures this is left for future work.

\section{Conclusions}

We have shown a way to derive a differential equation for the diffusion flux from the so-called mesoscopic theory. In this refined theory, an additional variable in the domain of the field quantities is introduced, here the velocity, and we have a velocity distribution. We have derived a differential equation for the diffusion flux from the mesocopic balance equations. The differential equation for the diffusion flux is of balance type, i.e., of the form used in extended thermodynamics. Therefore, it can be expected that our approach leads to hyperbolic systems of field equations, but the investigation of this question is left for future work.

Because the level here is not the microscopic one, no assumptions about interparticle interactions have to be made here in contrast to kinetic theory. We are here on the continuum level, and many-particle interactions are taken into account automatically. This is not the case for derivations of the field equations of extended thermodynamics based on the Boltzmann equation. 


\section{Acknowledgements}

We are grateful to W. Muschik for stimulating discussions and remarks. This work was supported by Fondi P.R.A 2002, University of Messina, Italy.

\section{References}

[1] Jou, D., Casas-Vazquez, J., Lebon, G., Extended Irreversible Thermodynamics, Springer-Verlag, Berlin, 1993.

[2] Jou, D., Casas-Vazquez, J., Lebon, G., Extended irreversible thermodynamics: An overview of recent bibliography, J. Non-Equilib. Thermodyn., 17 (1992), 383-396.

[3] Jou, D., Casas-Vazquez, J., Lebon, G., Recent bibliography on extended irreversible thermodynamics and related topics (1992-1995), J. Non-Equilib. Thermodyn., 21 (1996), 103-121.

[4] Jou, D., Casas-Vasquez, J., Lebon, G., Extended irreversible thermodynamics, Rep. Prog. Phys., 51 (1988), 1105-1179.

[5] Mueller, I., Ruggeri, T., Extended Thermodynamics, volume 37. Springer Tracts in Natural Philosophy, Springer, Berlin, 1993.

[6] Depiteux, N., Lebon, G., An extended thermodynamics modelling of nonFickian diffusion, J. Non-Newtonian Fluid Mech., 96 (2001), 105-117.

[7] Goldstein, P., Garcia-Colin, L.S., Transport processes in a viscoelastic binary mixture, J. Non-Equilib. Thermodyn., 19 (1994), 170-183.

[8] Goldstein, P., Garcia-Colin, L.S., A thermodynamic basis for transport phenomena in viscoelastic fluids, J. Chem. Phys., 99 (1993), 3913.

[9] Jou, D., Casas-Vazquez, J., Criado-Sancho, M., Polymer solutions under flow: Phase separation and polymer degradation, Adv. Polym. Sci., 120 (1995), 205266.

[10] Lebon, G., Jou, D., Casas-Vazquez, J., Nonequilibrium entropy and the second law of thermodynamics: A simple illustration, Int. J. Thermophys., 14 (1993), 671-683.

[11] Casas-Vazquez, J., Criado-Sancho, M., Jou, D., Dynamical and thermodynamical approaches to phase separation in polymer solutions under flow. Europhys. Lett., 23 (1993), 469-474.

[12] Criado-Sancho, M., Jou, D., Casas-Vazquez, J., On the spinodal line of polymer solutions under shear, J. Non-Equilib. Thermodyn., 18 (1992), 103120.

[13] Lebon, G., Casas-Vazquez, J., Criado-Sancho, M., Jou, D., Polymer solutions and chemical reactions under flow: A thermodynamic description, J. Chem. Phys., 98 (1993), 7434-7439.

[14] Mueller, I., Thermodynamics, Pitman Advanced Publishing Program, Boston, 1985.

[15] Mueller, I., Extended thermodynamics of classical and degenerate gases, Arch. Rat. Mech. Anal., 83 (1983), 286-332.

[16] Cimmelli, V.A., Ván, P., The effects of non-locality on the evolution of higher order fluxes in non-equilibrium thermodynamics, cond-mat/0409254, 2004. http://arxiv.org/PS_cache/cond-mat/pdf/0409/0409254.pdf 
[17] Ciancio, V., Cimmelli, V.A., Ván, P., Balance laws for higher order fluxes in non-equilibrium thermodynamics, cond-mat/0407530, 2005. http://arxiv.org/ PS_cache/cond-mat/pdf/0407/0407530.pdf

[18] Ruggeri, T., Symmetric-hyperbolic system of conservative equations for a viscous heat conducting fluid, Acta Mechanica, 77 (3) (1983), 167-183, Review Article.

[19] Weiss, W., Zur Hierarchie der Erweiterten Thermodynamik. Thesis. TU Berlin, Berlin, 1990.

[20] Friedrichs, K.O., Lax, P.D., Systems of conservation equations with a convex extension, Proc. Nat. Acad. Sci. USA, 68 (1971), 1686-1688.

[21] Lax, P.D., Hyperbolic Systems of Conservation Laws and the Mathematical Theory of Shock Waves, Society for Industrial and Applied Mathematics, Bristol, 1973.

[22] Born, M., Green, H.S., Proc. Roy. Soc. Lond. A, 188 (1946), 10.

[23] Born, M., Green, H.S., Proc. Roy. Soc. Lond. A, 190 (1947), 455.

[24] Bogoljuboff, N., J. Phys. USSR, 10 (1946), 265.

[25] Kirkwood, J.G., J. Chem. Phys., 14 (1946), 180.

[26] Muschik, W., Ehrentraut, H., Papenfuss, C., Blenk, S., Mesoscopic theory of liquid crystals, in: 25 Years of Non-Equilibrium Statistical Mechanics, Proceedings of the XIII Sitges Conference, 13-17 June 1994, Sitges, volume 445 of Lecture Notes in Physics, Eds. J.J. Brey, J. Marro, J.M. Rubi, M. San Miguel, pp. 303-311. Springer, Berlin, 1995.

[27] Muschik, W., Papenfuss, C., Ehrentraut, H., Concept of mesoscopic continuum physics with application to biaxial liquid crystals, J. Non-Equilib. Thermodyn., 25 (2000), 179-197.

[28] Papenfuss, C., Ciancio, V., Rogolino, P., Application of the mesoscopic theory to dipolar media, Technische Mechanik, 22 (2002), 132-140.

[29] Muschik, W., Papenfuss, C., Ehrentraut, H., Concepts of Continuum Thermodynamics. Kielce University of Technology, Technische Universität Berlin, ISBN 83-905132-7-7, 1996.

[30] Papenfuss, C., Muschik, W., Liquid crystal theory as an example of mesoscopic continuum mechanics, in: Trends in Continuum Physics, Eds. B.T. Maruszewski, W. Muschik, and A. Radowicz, pp. 277-291, World Scientific, Singapore, 1998.

[31] Blenk, S., Muschik, W., Orientational balances for nematic liquid crystals, J. Non-Equilib. Thermodyn., 16 (1991), 67-87.

[32] Blenk, S., Ehrentraut, H., Muschik, W., Macroscopic constitutive equations for liquid crystals induced by their mesoscopic orientation distribution, Int. J. Engng. Sci., 30 (9) (1992), 1127-1143.

[33] Blenk, S., Ehrentraut, H., Muschik, W., Statistical foundation of macroscopic balances for liquid crystals in alignment tensor formulation, Physica A, 174 (1991), 119-138.

[34] Ehrentraut, H., Hess, S., Viscosity coefficients of partially aligned nematic and nematic discotic liquid crystals, Phys. Rev. E, 51 (3) (1995), 2203-2212.

[35] Ehrentraut, H., Muschik, W., Papenfuss, C., Mesoscopically derived orientation dynamics of liquid crystals, J. Non-Equilib. Thermodyn., 22 (1997), 285298.

[36] Muschik, W., Ehrentraut, H., Papenfuss, C., The connection between ericksenleslie equations and the balances of mesoscopic theory of liquid crystals, Mol. Cryst. Liq. Cryst., 262 (1995), 417-423. 
[37] Van, P., Papenfuss, C., Muschik, W., Mesoscopic dynamics of microcracks, Phys. Rev. E, 62 (2000), 6206-6215.

[38] Ván, P., Papenfuss, C., Muschik, W., Griffith cracks in the mesoscopic microcrack theory. Published online: Condensed Matter, abstract, cond-mat/ 0211207; published J. Phys. A, 37 (2004), 5315-5328. http://arxiv.org/ PS_cache/cond-mat/pdf/0211/0211207.pdf

[39] Ehrentraut, H., A Unified Mesoscopic Continuum Theory of Uniaxial and Biaxial Liquid Crystals, Wissenschaft und Technik Verlag, Berlin, 1996.

[40] Blenk, S., Ehrentraut, H., Muschik, W., Orientation balances for liquid crystals and their representation by alignment tensors, Mol. Cryst. Liq. Cryst., 204 (1991), 133-141.

[41] Papenfuss, C., Theory of liquid crystals as an example of mesoscopic continuum mechanics, Comput. Mater. Sci., $19(2000), 45-52$.

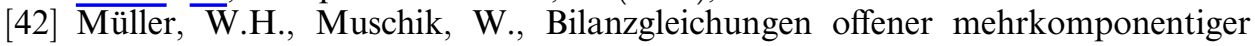
Systeme, I. Massen- und Impulsbilanzen, J. Non-Equilib. Thermodyn., 8 (1983), 29-46.

[43] Muschik, W., Müller, W.H., Bilanzgleichungen offener mehrkomponentiger Systeme, II. Energie- und Entropiebilanz, J. Non-Equilib. Thermodyn., 8 (1983), 47-66.

[44] Blenk, S., Ehrentraut, H., Muschik, W., A continuum theory for liquid crystals describing different degrees of orientational order, Liq. Cryst., 14 (1993), 1221 1226.

[45] Papenfuss, C., Muschik, W., Orientational order in free standing liquid crystalline films and derivation of a closure relation for higher order alignment tensors, Mol. Cryst. Liq. Cryst., 330 (1999), 541-548.

[46] Papenfuss, C., Nonlinear dynamics of the alignment tensor in the presence of electric fields, Arch. Mech., 50 (1998), 529-536.

Paper received: 2005-02-15

Paper accepted: 2005-04-27 
Bereitgestellt von | Technische Universität Berlin 